\title{
Mitral E/A Ratio
}

National Cancer Institute

\section{Source}

National Cancer Institute. Mitral E/A Ratio. NCI Thesaurus. Code C127571.

The ratio of the rate of blood flow during early diastole to the rate of blood flow during late diastole. 